\title{
Management of anaemia in patients with inflammatory bowel disease - results of a questionnaire among Polish healthcare professionals
}

\author{
Edyta M. Tulewicz-Marti ${ }^{1}$, Konrad Lewandowski ${ }^{1}$, Martyna Szczubełek ${ }^{1}$, Grażyna Rydzewska ${ }^{1,2}$ \\ ${ }^{1}$ Clinical Department of Internal Medicine and Gastroenterology with Inflammatory Bowel Disease Subdivision, \\ The Central Clinical Hospital of the Ministry of the Interior Affairs and Administration, Warsaw, Poland \\ ${ }^{2}$ Collegium Medicum, Jan Kochanowski University, Kielce, Poland
}

Gastroenterology Rev 2021; 16 (1): 89-94

DOI: https://doi.org/10.5114/pg.2021.104738

Key words: iron deficiency anaemia, iron deficiency anaemia, inflammatory bowel disease, ulcerative colitis, Crohn's disease, iron therapy.

\begin{abstract}
Address for correspondence: Edyta Maria Tulewicz-Marti MD, The Clinical Department of Internal Medicine and Gastroenterology with Inflammatory Bowel Disease Subdivision, The Central Clinical Hospital of the Ministry of the Interior Affairs and Administration, Warsaw, Poland, phone: +48 2250812 40, e-mail: e.tulewicz@gmail.com
\end{abstract}

\begin{abstract}
Introduction: Anaemia is the most common systemic and extraintestinal complication of inflammatory bowel disease (IBD). Its impact on quality of life is significant; hence, it is important for healthcare professionals to manage it correctly.

Aim: To assess the knowledge among doctors about the diagnostics and treatment of anaemia in IBD patients.

Material and methods: The questionnaire survey was conducted among 169 doctors of different specialties. Eighty-seven (51.5\%) of the respondents were gastroenterologists.

Results: $97.7 \%(84)$ of gastroenterologists and $92.6 \%$ (75) of all responders replied that all IBD patients should be monitored for anaemia $(p=0.266)$; however, only one-third of gastroenterologists knew the exact haemoglobin cut-off level in men with Crohn's disease. The necessity of monitoring vitamin $B_{12}$ was indicated by $53.7 \%$ (36) of gastroenterologists and by $24.1 \%(13)$ of other specialists $(p=0.002)$. Nine percent (6) of gastrologists and $3.7 \%(2)$ of other specialists screened for folic acid ( $p=$ 0.0431). $13.1 \%$ (11) of gastroenterologists and $35 \%$ (28) of other specialists frequently used iv iron $(p=0.003) .44 .1 \%(26)$ of gastroenterologists and 52\% (26) of other specialists administered between $1000 \mathrm{mg}$ and $1500 \mathrm{mg}$ of iv iron during hospitalization. Only $11.9 \%$ (7) of GI-specialists and 2\% (1) of non-Gl-specialists administered total doses over $1500 \mathrm{mg}(p=0.155) .71 \%$ (62) of gastroenterologists and 73\% (60) of all physicians did not observe any adverse events of iv iron.

Conclusions: Although the diagnostic approach to anaemia in IBD patients varies among respondents, knowledge of guidelines was slightly better among Gl-professionals then among other doctors.
\end{abstract}

\section{Introduction}

Anaemia is the most common systemic and extraintestinal complication of inflammatory bowel disease (IBD), which has a great impact on the patient's quality of life. It affects various aspects such as physical, emotional, and cognitive functions, work capacity, and frequency and duration of hospitalizations, and it generates costs for healthcare [1-3]. There are 2 main types of anaemia: iron deficiency anaemia (IDA) and anaemia of chronic disease (ACD). According to the WHO definition, which also applies to patients with IBD, anaemia is defined in women as haemoglobin $(\mathrm{Hb})$ level $<12.0 \mathrm{~g} / \mathrm{dl}$ and in men $<13.0 \mathrm{~g} / \mathrm{dl}$ [4]. Laboratory screening for IBD includes complete blood count, ferritin, and C-reactive protein levels. The frequency of these tests depends on the activity of the disease. Patients at risk of vitamin $B_{12}$ and folate deficiency (small intestine disease and after bowel resection) require additional attention. They should be tested at least once a year, and even more often when macrocytosis occurs. Iron supplementation is recommended for any patient who is diagnosed with iron deficiency anaemia, and its diagnostic and therapeutic procedures should be implemented when the haemoglobin level decreases. Oral iron supplementation is justified in patients with mild anaemia; however, the first-line treatment is intravenous iron supplementa- 
tion $[5,6]$. The goal of replenishing iron deficiency is to maintain normal haemoglobin and ferritin levels but even with intensive treatment, anaemia can return quickly. It is reasonable to prevent it and maintain adequate levels of iron and vitamins. It is crucial for healthcare professionals to correctly manage anaemia in IBD patients.

\section{Aim}

Therefore, the main purpose of the survey was to assess physicians' knowledge of the guidelines for anaemia management in IBD patients.

\section{Material and methods}

An anonymous questionnaire with 13 questions was conducted during a Polish conference among medical doctors interested in gastrointestinal (GI) tract diseases, such as gastroenterologists, physicians, surgeons, and medical doctors without any specialty. The questionnaire, in Polish language, assessed the diagnostic criteria and therapeutic modalities for anaemia for each respondent. The survey included data concerning place of employment, number of patients the doctor treated, and questions about appropriate diagnostic tools and optimal therapeutic interventions. The responses were evaluated based on European Crohn's and Colitis Organization (ECCO) guidelines and Polish Society of Gastroenterology guidelines for the diagnosis and treatment of anaemia in IBD.

\section{Statistical analysis}

Comparisons between groups of doctors were done using the $\chi^{2}$ test or exact Fisher test (in case of less frequent answers). Analysis was carried out using R 3.6.3 statistical software.

\section{Results}

The total number of participants was 169 . Eightyseven $(51.5 \%)$ respondents were gastroenterology specialists. The remaining participants were specialists in internal medicine - 48 (28.4\%) people, and 28 (16.6\%) doctors undergoing specialist training. The least numerous group of doctors were specialists in surgery $6(3.6 \%)$ participants.

A significant majority of participants (123 (72.8\%)) reported that their current work setting was at a university hospital, and the remaining 50 (29.6\%) practiced in voivodship and county hospitals. Among professionals working in clinical hospitals, the survey included 69 (57.5\%) gastroenterologists, 29 (24.2\%) internal diseases specialists, 4 surgeons, and 18 doctors in specialization training (15.0\%). In non-university hospitals, the largest number of doctors were internal medicine - 19 (38.8\%), then there were $18(36.7 \%)$ gastroenterologists and $2(4.1 \%)$ surgeons $(p=0.102)$. For the purposes of the following survey, we compared the responses of gastroenterologists (87 doctors) with specializations other than gastroenterology (82 doctors).

Respondents were asked to fill in the anonymous questionnaire. Figure 1 shows that over $94 \%$ of all doctors knew the importance of screening of IDA in IBD, but less than $30 \%$ knew the exact level of haemoglobin in $\mathrm{CD}$ in male and female patients. Moreover, $8.9 \%$ of physicians knew the laboratory parameters needed for screening of IDA. Over $60 \%$ of doctors knew the most effective parameter in IDA screening.

As shown in Figure 2, a significant majority of gastroenterologists (84 (97.7\%) physicians) replied that all patients should be monitored for anaemia and $2(2.33 \%)$ physicians replied that monitoring requires exacerbation of patients. Among other doctors $75(92.6 \%)$ marked the correct answer $(p=0.266)$.

Regarding the haemoglobin concentration in women with Crohn's disease requiring treatment (the correct answer was $12 \mathrm{~g} / \mathrm{dl}$ ), less than $30 \%$ of all respondents gave the correct answer. It was given by 30 (34.9\%) GI specialists. Among physicians other than gastroenterologists, $18(22 \%)$ respondents gave the correct response $(p=0.158)$.

Twenty-five (29.1\%) gastroenterologists knew the cut-off level of haemoglobin in men with Crohn's disease requiring treatment; however, most of them chose $12 \mathrm{~g} / \mathrm{dl}$ (28 (32.6\%) doctors). Among physicians other than gastroenterologists, 17 (20.7\%) people gave the correct answer. No statistical significance was obtained in the question ( $p=0.473)$.

Concerning parameters to diagnose anaemia in their daily practice (complete blood count, C-reactive protein level, and ferritin) in patients with IBD, only $6(7.3 \%)$ gave the correct answer among gastrologists and 9 (11.5\%) among doctors of other specializations ( $p=0.519)$.

The next issue was the frequency of determination of iron metabolism parameters in patients with ulcerative colitis in clinical remission. Among gastroenterologists, most physicians (77 (88.5\%) doctors) chose the correct answer of every 6-12 months, while 10 (11.5\%) doctors replied every 3 months. Seventy-three (89\%) doctors gave the correct answer among other specialties. Nine (11\%) people indicated that iron parameters should be determined every 3 months $(p=0.066)$.

Regarding patients with Crohn's disease after ileal resection, 36 (53.7\%) gastroenterologists and 13 $(24.1 \%)$ other doctors reported that vitamin $B_{12}$ levels should be measured $(p=0.002)$. However, only $2(3.7 \%)$ 


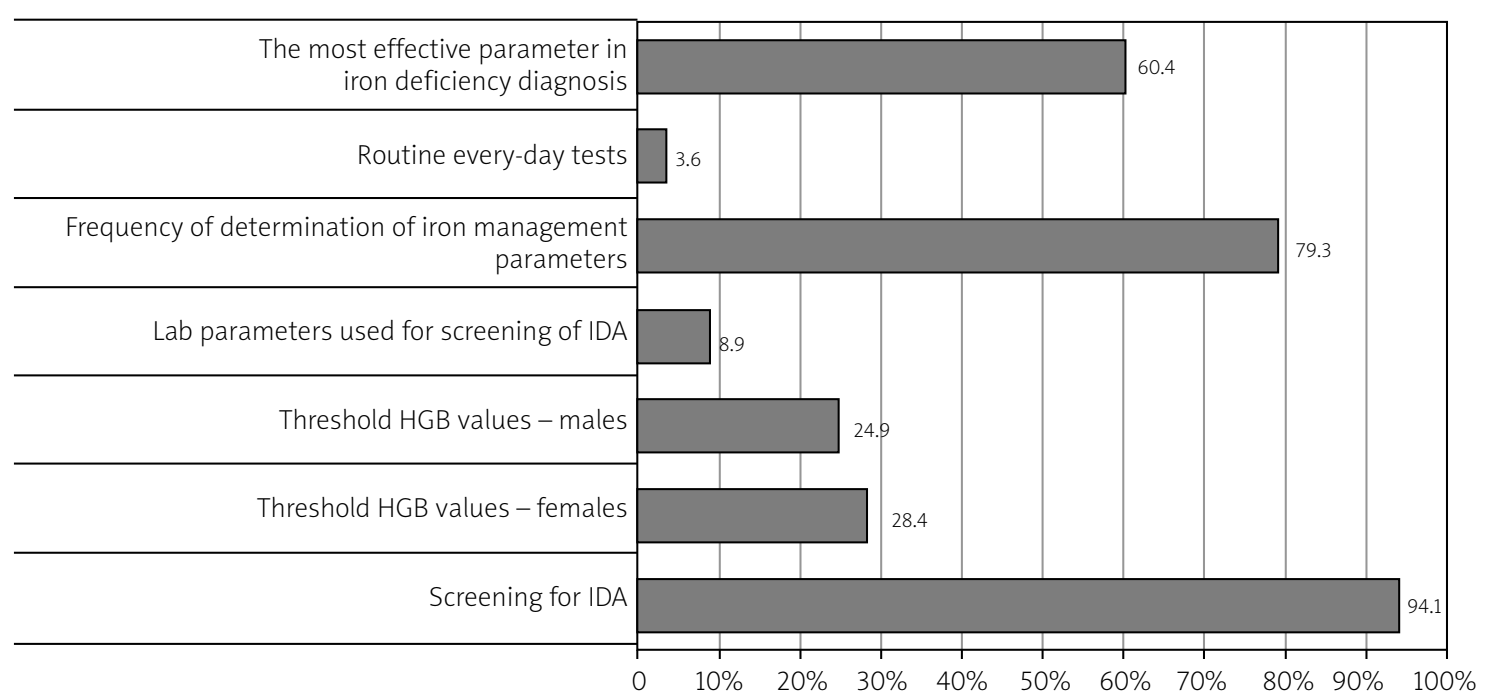

Figure 1. Correct answers of survey responded by all healthcare specialists (gastroenterologists and non-gastroenterologists)

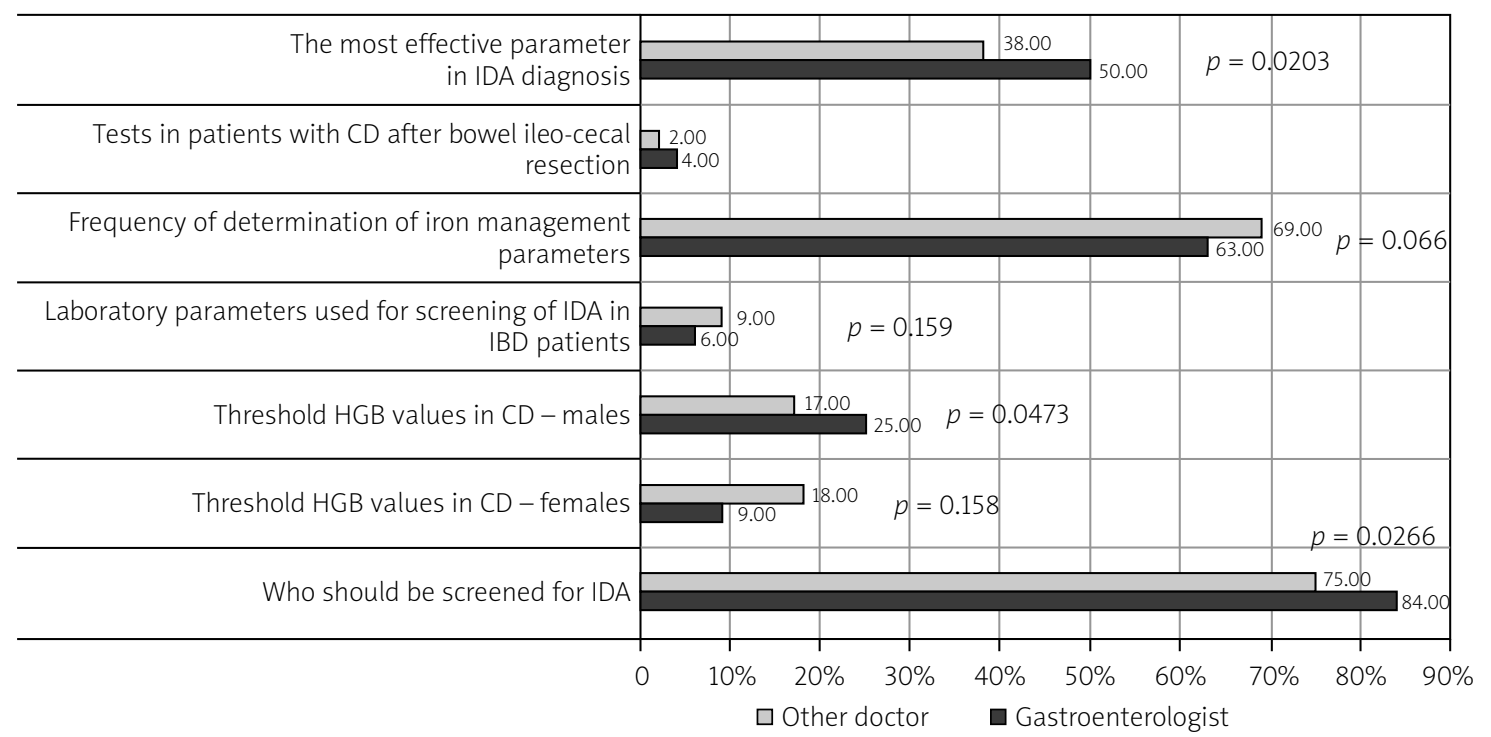

Figure 2. Responses concerning managing anemia in IBD answered by specialties- gastroenterologists and other specialty doctors

and $6(9 \%)$ gastroenterologists reported the same about folate acid $(p=0.321)$.

Fifty (60\%) gastroenterologists and 38 (47.5\%) other specialists knew that the most useful parameter in the diagnosis of IDA in IBD patients is the ferritin level $(p=0.203)$.

Figure 3 shows the next part of the questionnaire, concerning iron treatment. The most commonly used iron formula was oral treatment - 69 (82.1\%) gastroenterologists. Fifty (62.5\%) other doctors chose this option. Eleven (13.1\%) gastrologists and 28 (35\%) other specialists used the iv formula. One (1.2\%) gastroenterologist and 2 (2.5\%) other doctors prescribed intramus- cular iron preparation, and 3 (3.6\%) gastroenterologists used both vo and iv iron $(p=0.003)$.

Respondents were asked how many patients per month were treated with intravenous iron; their answers were 0 to 30 patients per month. Twenty-two (27\%) Gl-specialists and 4 (5\%) doctors of other specialties treated over 30 patients per month. Fifty-three percent of other physicians applied iv iron in less than 5 patients per month $(p=0.001)$.

Regarding the maximum iron dose during hospitalization, the most commonly recommended dose by all specialists was between $1000 \mathrm{mg}$ and $1500 \mathrm{mg}$ (26 $44 \%$ among gastroenterologists and $26-52 \%$ among 
other specialists). Twenty-eight percent (14) of respondents among other specialists and 34\% (20) among Gl specialists used between $500 \mathrm{mg}$ and $1000 \mathrm{mg}$. Fourteen percent (7) and 5\% (3) of respondents used low doses of less than $500 \mathrm{mg}$. Four percent (2) of doctors and 5\% (3) of GI specialists used the Ganzoni formula to calculate the dose of iron.

The maximum iv iron daily dose between 500 and $1000 \mathrm{mg}$ was applied by $47 \%$ (22) of other doctors and by $51 \%$ (29) of gastroenterologists. Thirty-two percent (15) of other specialists gave $500 \mathrm{mg}$ iron iv, as did 23\% (13) of gastroenterologists. A dose between 1000 and $1500 \mathrm{mg}$ was prescribed by $21 \%$ (10) and $23 \%$ (13) of Gl specialists $(p=0.465)$

Unfortunately, only $44 \%$ of GI specialists repeated iv iron infusions. Other specialists gave ambiguous answers: $50 \%$ repeated iron infusions and 50\% did not $(p=0.563)$.

Regarding the side effects of iv iron, the majority of surveyed doctors (71\% among gastroenterologists and $73 \%$ among other doctors) did not observe any adverse effects $(p=0.917)$.

\section{Discussion}

Despite anaemia being the most prevalent extraintestinal manifestation of IBD and its most frequent systemic complication with a great impact on the quality of life of IBD patients, it may easily be misdiagnosed. What is more, a high rate of anaemia is one of the measurements of quality of care of IBD patients [7]. The aim of our study was to evaluate the knowledge of medical doctors about the diagnosis and treatment of anaemia in IBD patients. The results suggest that there is still room for improvement in its education.

ECCO and Polish Society of Gastroenterology guidelines outline that IBD patients should be regularly assessed for the presence of anaemia. Figure 1 shows that $94.1 \%$ of respondents know that all patients with IBD should be screened for anaemia. In this group better answers were given by Gl specialists ( $p=0.027)$. When it comes to detailed knowledge, less than a third of respondents knew the exact low limit of haemoglobin concentration in Crohn's disease to start treatment in women $(p=0.158)$ and men $(p=0.047)$. This knowledge was slightly better in GI specialists. Only $8.9 \%$ of respondents knew the exact parameters used for screening of IDA in IBD patients, and in this group other specialists gave good answers $(p=0.159)$.

It is fundamental to know that in the majority of cases anaemia is a combination of iron deficiency (IDA) and chronic disease (ACD), but there are other factors such as vitamin $B_{12}$ and folate deficiency, toxic effects of medication, etc., which may influence its heterogeneity. According to the diagnostic approach of anaemia, laboratory screening, including complete blood count, serum ferritin, and C-reactive protein (CRP), should be performed every 6-12 months for patients in remission or with mild disease, and for those with active disease - every 3 months.

Among all respondents, only $7.3 \%$ of gastroenterologists and $11.5 \%$ of doctors with other specialties knew the exact laboratory screening parameters of anaemia $(p=0.519)$. The vast majority of all respondents knew the frequency of measurements in IDA in CU. In regard

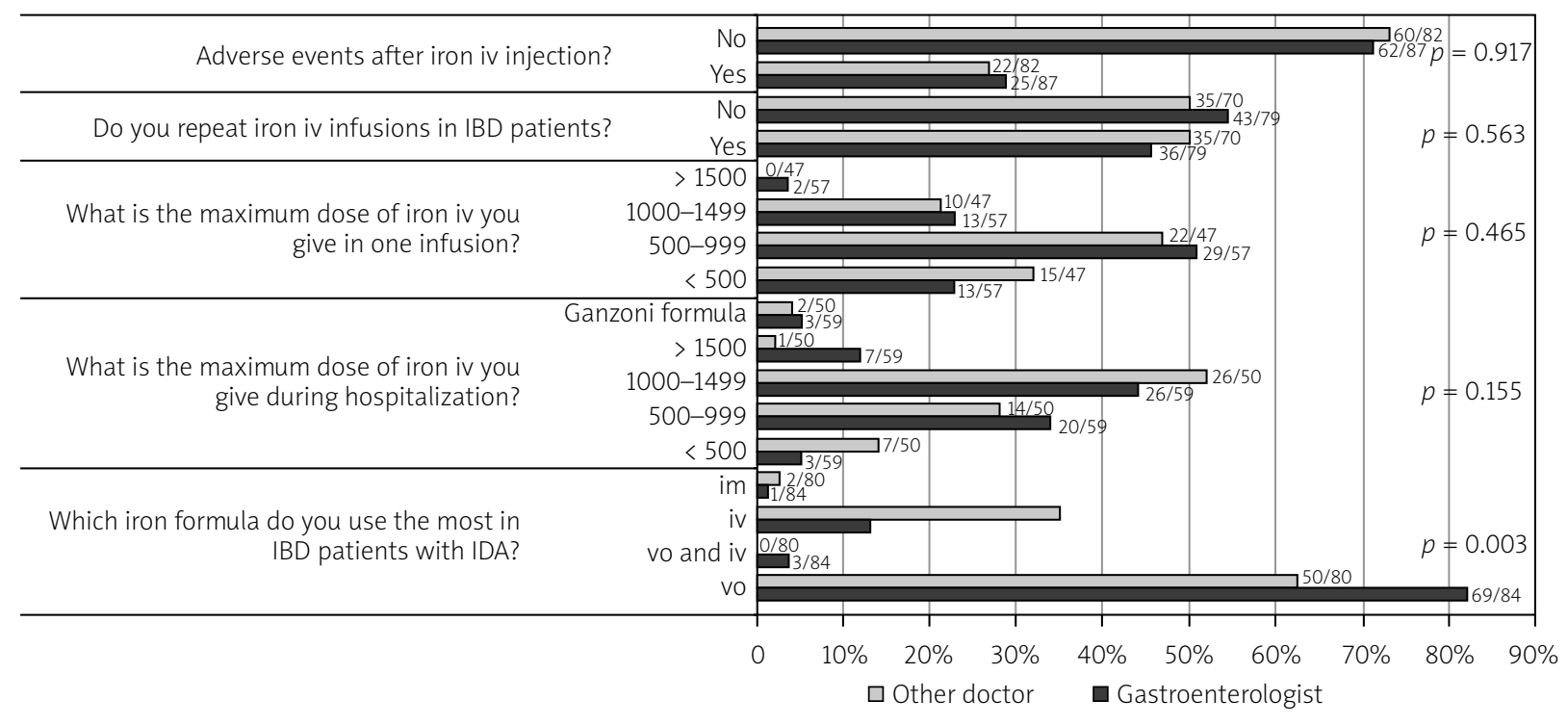

Figure 3. Responses concerning iron treatment by specialties- gastroenterologists and other specialty doctors 
to patients in the risk group for vitamin $\mathrm{B}_{12}$ and folic deficiency (small bowel disease or ileal resection), $53.7 \%$ of gastroenterologists and $24.1 \%$ of other doctors reported that vitamin $B_{12}$ levels should be measured ( $p=0.002$ ). Only $9 \%$ of gastroenterologists and $3.7 \%$ of other doctors remembered about folate measurement $(p=0.321)$. As for the best screening parameter in diagnosis of IDA, it is essential to know that it is ferritin; the correct answer was given by $62 \%$ of gastroenterologists and by $72 \%$ of other specialists ( $p=0.020$ ).

According to our study, the majority of respondents (82.0\% of Gl-specialists and $62.5 \%$ of other doctors) prescribed oral iron ( $p=0.003)$. Oral formula can be effective only under specific circumstances and may be used in patients with mild anaemia and clinically inactive disease, with no previous oral iron intolerance. Luminal iron changes the microbiota profile and bacterial metabolism $[8,9]$. Interestingly, in animal models of colitis, oral iron may exacerbate colitis and promote carcinogenesis [10, 11]. Furthermore, duodenal disease involvement may impair iron absorption, and elevated circulating hepcidin may block the release of iron taken up by enterocytes into circulation [12]. Hence, according to ECCO and Polish Guidelines, intravenous iron should be the first-line treatment in patients with clinically active IBD, with previous intolerance to oral iron and haemoglobin below $10 \mathrm{~g} / \mathrm{dl}$, and in patients who need erythropoiesis-stimulating agents. The intravenous iron preparations are safe and well tolerated and bypasses the sites of inflammation. However, few gastroenterologists use iv iron (Figure 3). The most frequent iv iron formulations are ferric carboxymaltose and ferric derisomaltose (known as iron [III] isomaltoside). A single dose for ferric carboxymaltose infusion is up to $20 \mathrm{mg}$ iron/kg body weight and should not exceed $1000 \mathrm{mg}$ of iron per week. A single dose for ferric derisomaltose infusion is up to $20 \mathrm{mg}$, without a single dose additional limit. For the majority, especially young and working patients, having the possibility of effective single dose intravenous therapy is very important [13]. The estimation of the dose of iron should be based on baseline haemoglobin and body weight. According to guidelines, a minimum of 500-1000 mg should be applied. Only $22.8 \%$ (13) of gastroenterologists and $31.9 \%$ (15) of other doctors prescribed low iron doses. The risk of iron overload in IBD patients with chronic bleeding is extremely low; however, ferritin above $800 \mu \mathrm{g} / \mathrm{l}$ should be the upper limit. $44.1 \%$ (36) of gastroenterologists and $50 \%$ (30) of other physicians repeated the iron dose. Moreover, in our study, $1.2 \%$ of the Gl specialists and 2.5\% used intramuscular iron; however, it is currently not recommended in IBD patients because of its many side effects.
Our results are comparable to the results of the survey study "Gestiona Hierro ElI" [14] about iron treatment in IBD patients, conducted among Spanish gastrologists. According to the Spanish survey $97 \%$ of respondents knew that it is essential to screen for anaemia in IBD patients, and over $70 \%$ of gastroenterologists used ferritin as a marker of anaemia. Hence, comparing to Polish gastroenterologists, the Spanish recommend CRP more often ( $90 \%$ vs. $13 \%$ ). Regarding iron treatment, also the majority of gastroenterologists started iron replacement from oral formula, then iv, and finally $4.9 \%$ of GI specialists chose iron im. Moreover, only $33.1 \%$ of Spanish gastroenterologist use high (more than $1000 \mathrm{mg}$ ) iron doses compared to Polish gastroenterologists who use high doses and repeat the iron if necessary.

This study has some limitations. First, the group of participants might not be sufficiently heterogenic (respondents were recruited during the conference). Moreover, the open questions might not have been clear to respondents. Nonetheless, to our knowledge, no prior Polish surveys regarding the treatment of anaemia in IBD patients have been published with the aim of improving its management.

\section{Conclusions}

Knowledge about the guidelines of treatment of anaemia in IBD patients is widely variable among healthcare professionals practicing in Poland, but it is slightly better among gastroenterologists. Management of patients with IDA in IBD, especially by GI-specialists, is satisfying; however, implementing the guidelines to everyday practice should ameliorate this care.

\section{Conflict of interest}

The authors declare no conflict of interest.

\section{References}

1. Vegh Z, Kurti Z, Gonczi L, et al. Association of extraintestinal manifestations and anaemia with disease outcomes in patients with inflammatory bowel disease. Scand J Gastroenterol 2016; 51: 848-54.

2. Koutroubakis IE, Ramos-Rivers C, Regueiro M, et al. Persistent or recurrent anemia is associated with severe and disabling inflammatory bowel disease. Clin Gastroenterol Hepatol 2015; 13: 1760-6.

3. Gasche C, Berstad A, Befrits R, et al. Guildelines on the diagnosis and management of iron deficiency and anemia in inflammatory bowel diseases. Inflamm Bow Dis 2007; 13: 1545-53.

4. WHO, UNICEF, UNU Iron Deficiency Anemia: Assessment, Prevention and Control. Report of a joint WHO/UNICEF/UNU consultation. Geneva. World Health Organization 1998.

5. Dignass AU, Gasche C, Bettenworth D, et al. European consensus on the diagnosis and management of iron deficiency 
and anaemia in inflammatory bowel diseases. J Crohns Colitis 2015; 9: 211-22.

6. Kaniewska M, Bartnik W, Gonciarz M, et al. Iron deficiency anaemia in patients with inflammatory bowel disease: National Consultant for Gastroenterology Working Group Recommendations. Prz Gastroenterol 2014; 9: 259-63.

7. Masuger W, Moore GTC, Andrews JM, et al. Crohn's \& Colitis Australia inflammatory bowel disease audit: measuring the quality of care in Australia. Intern Med J 2019; 49: 859-66.

8. Lee T, Clavel T, Smirnov K, et al. Oral versus intravenous iron replacement therapy distinctly alters the gut microbiota and metabolome in patients with IBD. Gut 2017; 66: 863-71.

9. Zimmermann MB, Chassard C, Rohner F, et al. The effects of iron fortification on the gut microbiota in African children: a randomized controlled trial in Cote d'Ivoire. Am J Clin Nutr 2010; 92: 1406-15.

10. Mahalhal A, Williams JM, Johnson S, et al. Oral iron exacerbates colitis and influences the intestinal microbiome. PLoS One 2018; 13: e0202460.

11. Seril DN, Liao J, Ho KL, et al. Dietary iron supplementation enhances DSS-induced colitis and associated colorectal carcinoma development in mice. Dig Dis Sci 2002; 47: 1266-78.

12. Jimenez KM, Gasche C. Management of iron deficiency anaemia in inflammatory bowel disease. Acta Haematol 2019; 142: 30-6.

13. Milovanovic S, Scaldaferri F, Canarecci S, et al. Therapy experiences and preferences among patients with anemia: results of a cross-sectional survey among Italian patients with inflammatory bowel disease. Dig Liver Dis 2017; 49: 1098-103.

14. Casellas Jordá F, Vera Mendoza I, Barreiro-de Acosta M, et al. Managing iron deficiency and iron deficiency anemia in inflammatory bowel disease. The results of the "Gestiona hierro-Ell” survey. J Rev Esp Enferm Dig 2018; 110: 172-8.

Received: 1.07 .2020

Accepted: 8.07.2020 